\title{
From In Situ Conversion to Chemical Reaction Kinetics: Development of Truly Operando TEM and its Application to CeO2-Supported Pt Catalysts
}

Joshua Vincent $^{1}$ and Peter Crozier ${ }^{2}$

${ }^{1}$ Arizona State University, United States, ${ }^{2}$ Arizona State University, Tempe, Arizona, United States

Chemical and electrochemical processes play a critical role in the existing and future energy technologies. However, many promising clean energy conversion and storage solutions remain limited by the lack of catalysts with suitable activity, stability, and selectivity. In order to improve the performance of catalysts in these applications, an understanding must be attained as to how the atomic-level structure of the catalyst surface regulates the kinetics of the chemical reaction of interest. Doing so is considerably challenging. Catalytically significant surface structures are known to emerge and transform during catalysis, requiring characterization techniques that provide information from controlled environments, i.e., in situ under reaction conditions [1]. Aberration-corrected in situ environmental transmission electron microscopy (ETEM) is a powerful tool capable of providing atomic-level information from high surface area catalysts. In the last decade there has been considerable advancements in methodology and instrumentation for studies under working conditions, including in-line gas analysis (e.g., spectroscopy or mass spectrometry) that allows the gas composition in or downstream from the reaction cell to be monitored. Measurements of the gas composition can confirm that catalysis is actually taking place, and in favorable cases they may be used to quantify the in situconversion of reactants to products.

An aspirational goal for in situ ETEM research would be to establish a direct link between the observed structure and the functionality (e.g., activity) of the catalytic material. It is important to stress that the in situ conversion is not itself and in many times not simply related to the activity of the working catalyst. The activity of the catalyst is better expressed in terms of a mass, surface area, or site-normalized reaction rate. However, heat and mass transport gradients in the in situreaction cell can limit the kinetics of the reaction and cause the activity of the catalyst to appear lower than it actually is. In the field of reaction engineering, this knowledge has led to the design of carefully controlled packed-bed catalytic reactors operated under so-called differential conditions, where the conversion can be directly related to the kinetics of the catalyzed chemical reaction. The latter quantity reflects the true activity of the catalyst, and when measured in situ under reaction conditions during a simultaneous measurement of the catalyst's structure, the experiment may be called operando $[2,3]$. Terminology aside, the distinction has important scientific implications: since conversion is not directly relatable to kinetics except under ideal circumstances, correlating structural information with conversion alone could yield misleading conclusions about the nature of structure-activity relationships.

The motivation of the present work is to develop and apply quantitative operando methods for open cell ETEM. We have performed extensive modeling of the ETEM reactor developed by our group in which a catalyst-loaded pellet is loaded into a furnace-based hot stage along with a metal mesh for atomicresolution imaging $[4,5]$. The model provides a foundation for kinetic analysis, allowing the conversion measured with electron energy-loss spectroscopy to be related to the reaction rate of the catalyst that is imaged on the TEM grid [6]. We have applied this methodology to elucidate structure-activity relationships in $\mathrm{Pt} / \mathrm{CeO}_{2}$ catalysts performing $\mathrm{CO}$ oxidation. Figure 1 shows a summary of the experimental and theoretical kinetic analysis, including (a) detection of product $\mathrm{CO}_{2}$ and (b) quantification of in situ CO conversion, which shows good agreement with the calculations. Part (c) shows the simulated 3D temperature distribution for a furnace set point of $275{ }^{\circ} \mathrm{C}$. Finally, in part (d) we show that the reaction rate of particles on the TEM grid (solid blue line) can be calculated from the rate estimated 
using the EELS CO conversion (dashed red line) by scaling the estimated value by a factor of 1.45 . The mass of catalyst loaded in the reactor and an estimate of the number of perimeter sites has been made, enabling the rate to be determined on a site-normalized turnover frequency (TOF) basis. Figure 2 displays a series of 12.5 second time-averaged operando TEM images of the $\mathrm{CeO}_{2}$-supported $\mathrm{Pt}$ nanoparticles during CO oxidation for TOFs of (a) 0.00 , (b) 0.80 , and (c) $1.05 \mathrm{CO} \mathrm{site} \mathrm{sec}^{-1}$. The increasing frequency of catalytic turnover is seen to correlate with dynamic structural behavior which can be attributed to an enhanced rate of oxygen vacancy creation and annihilation. At the same time, the $\sim 1.5 \mathrm{~nm}$ Pt nanoparticles become increasingly destabilized and undergo continuous and more frequent fluxional behavior. The results demonstrate that the bonding environment at the interface is constantly changing during catalysis, and they implicate the interfacial Pt-O-Ce bonds anchoring the Pt to the support as being involved also in the catalytically-driven oxygen transfer process $[7,8]$.
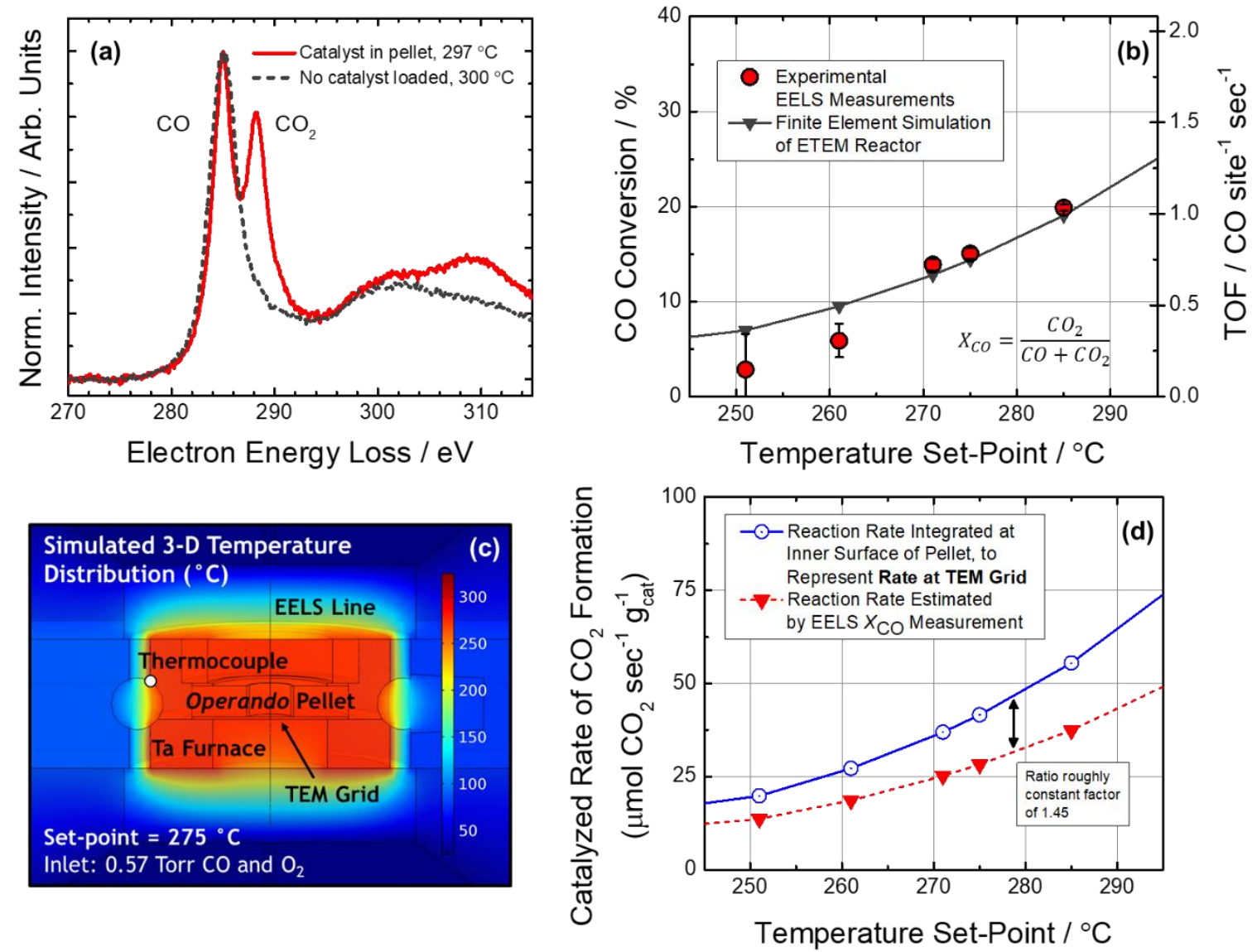

Figure 1. Detection and quantification of in situ CO conversion and chemical reaction rate in the ETEM reactor. In (a) EEL spectra demonstrate the detection of catalytically-produced $\mathrm{CO} 2$. In (b) the $\mathrm{CO}$ conversion has been quantified and shows good agreement with simulation under nominally identical conditions. In (c) the simulated temperature profile in the ETEM reactor is shown for a furnace set point of $275^{\circ} \mathrm{C}$. Finally, in (d) an analysis of the catalyzed rate of product formation in the model is presented, showing that the relative difference between the rate estimated through the EELS CO conversion and the rate of catalyst imaged on the TEM grid is a roughly constant factor of 1.45 . 


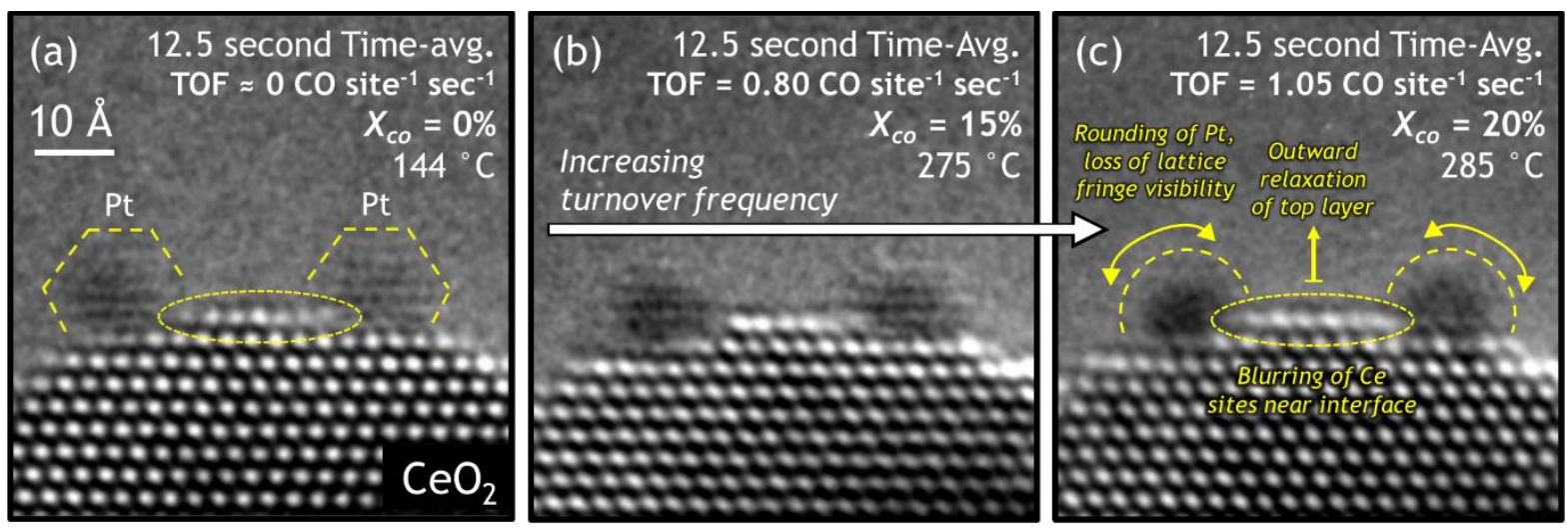

Figure 2. Time-averaged $12.5 \mathrm{sec}$ operando TEM images acquired during $\mathrm{CO}$ oxidation showing the dynamic structural evolution of the $\mathrm{Pt} / \mathrm{CeO} 2$ catalyst for TOFs of (a) $0.00 \mathrm{CO}$ site- 1 sec-1, (b) $0.80 \mathrm{CO}$ site-1 sec-1, and (c) 1.05 CO site-1 sec-1.

References

1. Tao, F. and Crozier, P. A., Chemical Reviews, 166 (2016), p. 3487-3539.

2. Bañares, M. A., Catalysis Today, 100 (2005), p. 71-77.

3. Guerrero-Pérez, O. M. and Bañares, M. A., Catalysis Today, 113 (2006), p. 48-57.

4. Chenna, S., and Crozier, P. A., ACS Catalysis, 2 (2012), p. 2395-2402.

5. Miller, B. K., Barker., T. M., and Crozier, P. A., Ultramicroscopy, 156 (2016), p. 18-22.

6. Vincent, J. L., et al., Ultramicroscopy, 218 (2020), 113080.

7. Vincent, J. L., and Crozier, P. A., manuscript in preparation.

8. We gratefully acknowledge NSF grant CBET-1604971 and the facilities at the John M. Cowley Center for High Resolution Electron Microscopy at Arizona State University. 